\title{
Enquête
}

Archives de la revue Enquête

1 | 1995

Les terrains de l'enquête

\section{La production de la théorie à partir des données}

The production of grounded theory

\section{Barney G. Glaser et Anselm L. Strauss}

Traducteur : Jean-Louis Fabiani

\section{OpenEdition}

\section{Journals}

Édition électronique

URL : http://journals.openedition.org/enquete/282

DOI : $10.4000 /$ enquete. 282

ISSN : 1953-809X

Éditeur :

Cercom, Éditions Parenthèses

Édition imprimée

Date de publication : 1 octobre 1995

Pagination : 183-195

\section{Référence électronique}

Barney G. Glaser et Anselm L. Strauss, "La production de la théorie à partir des données », Enquête

[En ligne], 1 | 1995, mis en ligne le 10 juillet 2013, consulté le 23 avril 2019. URL : http:// journals.openedition.org/enquete/282 ; DOI : 10.4000/enquete.282 


\title{
La production de la théorie à partir des données
}

The production of grounded theory

\author{
Barney G. Glaser et Anselm L. Strauss
}

Traduction : Jean-Louis Fabiani

On connait mieux depuis quelques années en France l'importance de l'œuvre d'Anselm Strauss ${ }^{1}$, surtout grâce au beau travail d'édition accompli par Isabelle Baszanger². Né en 1916 à New York, Strauss fait ses études de sociologie à l'université de Chicago dans les années quarante: Herbert Blumer et Everett Hugues sont alors ses "héros intellectuels", comme il aime à le rappeler. Il enseigne dans cette université dès 1952 et devient une des figures les plus remarquables, avec Erving Goffman, Howard Becker, Fred Davis et Eliott Freidson, du puissant mouvement de sociologie d'enquête qui se développe dans la postérité de la philosophie pragmatiste et des monographies pionnières de "l'école de Chicago ». D'une bibliographie abondante on a surtout retenu la contribution à l'interactionnisme, à travers les analyses des processus d'identification, la problématique de la négociation ou celle de la pluralité des mondes sociaux, ainsi que la préoccupation méthodologique: Anselm Strauss relève le défi que constitue l'exploitation rigoureuse d'un matériau "qualitatif » à partir de la description fine d'opérations de codage et de recodage dans de nombreux textes qui n'esquivent pas la dimension pédagogique. Mais on a moins bien perçu l'intérêt que présentent les propositions épistémologiques de Strauss. On a trop souvent considéré que les sociologues interactionnistes étaient des observateurs très fins mais qu'ils étaient aussi de "petites têtes" théoriques. The Discovery of Grounded Theory tend à montrer le contraire, et la traduction de l'extrait qui suit est une contribution à la réévaluation de l'épistémologie interactionniste. Sans aucune emphase, et en restant toujours au plus près des exigences spécifiques de l'observation réglée du monde social, Glaser et Strauss indiquent les impasses auxquelles conduisent les problématiques de la validation d'une théorie par les tests empiriques, comme celle de Merton: c'est à un autre régime de la production théorique qu'obéissent les sciences sociales, lesquelles ne satisfont pas aux conditions logiques de la "falsification ». Cette particularité ne conduit pas pour autant à l'abandon d'un usage exigeant de la notion de « théorie» : mais c'est un autre type de théorie que celui que retiennent ordinairement 
les épistémologies naturalistes appliquées aux sciences sociales. Si ce texte de 1967 est évidemment daté par ses références et son style, il n'en reste pas moins une contribution très convaincante à l'affirmation d'un espace épistémologique propre aux sciences sociales. On rapprochera utilement cet extrait des articles de Jean-Claude Passeron et de Jean-Pierre Olivier de Sardan publiés dans ce numéro.

JEAN-LOUIS FABIANI

La notion d'analyse comparative, d'usage fréquent en sociologie et en anthropologie, a été investie de significations différentes au cours de son développement et peut de ce fait constituer le support de tâches différentes. De nombreux sociologues et anthropologues, reconnaissant la grande puissance de l'analyse comparative, l'ont utilisée pour mener à bien des objectifs variés. Pour éviter la confusion, nous devons donc d'entrée de jeu clarifier l'usage que nous faisons de cette méthode : il s'agit de la production de théorie. Nous commencerons par distinguer cet usage de certains autres. Nous proposerons ensuite une définition et une description du type de théorie qui peut être engendré à partir de l'analyse comparative.

3 L'analyse comparative est une méthode de portée générale, au même titre que les méthodes expérimentale et statistique (toutes utilisent la logique de la comparaison). En outre, comme les deux autres méthodes, l'analyse comparative peut être utilisée pour des objets sociaux de n'importe quelle taille. Des sociologues et des anthropologues ont l'habitude d'utiliser le terme d'analyse comparative uniquement en référence à des comparaisons qui portent sur des objets sociaux de grande dimension, en particulier les organisations, les nations, les institutions, et de vastes régions du monde. Mais une telle référence restreint la portée de la méthode à une classe particulière d'objets sociaux auxquels elle a été le plus souvent appliquée. Notre discussion de l'analyse comparative comme méthode stratégique pour produire de la théorie lui confère sa généralité la plus complète en étendant sa validité à des objets sociaux de n'importe quelle taille, qu'elle soit grande ou petite, depuis les individus ou leurs rôles sociaux jusqu'aux nations ou aux grandes régions du monde. Notre expérience récente a fait la démonstration de l'utilité de cette méthode pour l'étude des petits objets sociaux organisés, comme des pavillons dans les hôpitaux ou des classes dans une école ${ }^{3}$.

4 Avant de distinguer notre objectif dans ce domaine d'autres objectifs, nous voudrions évoquer un usage malheureux de la comparaison : celle qui vise à démolir, à réfuter ou à disqualifier le travail de collègues. À partir de ses propres observations, un sociologue a presque toujours les moyens de trouver, s'il en a envie, des données qui réfutent le point d'appui empirique sur lequel son collègue a fondé une notion théorique. De nombreux sociologues le font! Si chaque démolisseur avait à l'esprit la valeur potentielle de l'analyse comparative, au lieu de satisfaire une envie pressante de "descendre " un collègue, il s'apercevrait qu'il vient seulement de constituer un autre datum comparatif susceptible d'engendrer une autre propriété ou une autre catégorie théorique. Il n'a pas fait autre chose. Rien n'est réfuté ni démoli, en dépit de ce que croient constamment ceux qui font montre d'une préoccupation excessive pour la preuve. Des collègues mieux disposés, qui signalent au sociologue un ou plusieurs cas négatifs mais qui craignent d'affaiblir sa motivation, auront généralement pour attitude de lui suggérer que son assertion théorique devrait faire l'objet d'une restriction ou d'une requalification. 
L'analyse comparative qu'ils proposent aide le chercheur à reparcourir sa propre analyse et à aller plus loin dans sa propre production théorique.

5 Nous voudrions aussi engager le dialogue avec ceux qui "descendent» la stratégie comparative en disant qu'elle n'est pas "particulièrement originale». Il est vrai que la notion générale d'analyse comparative a été développée par les pères fondateurs de la sociologie - Weber, Durkheim, Mannheim - et par l'anthropologie sociale. Nous pouvons seulement espérer que nos lecteurs seront suffisamment attentifs aux détails de l'analyse comparative que nous présentons pour être en mesure de repérer les avancées stratégiques qui conduisent à un usage complètement renouvelé de la notion.

\section{Les objectifs de l'analyse comparative}

6 La distinction que nous avons faite à propos du poids relatif de la production d'assertions théoriques et de la vérification peut trouver une illustration supplémentaire si l'on considère les usages typiques de la preuve qu'on obtient par l'analyse comparative.

\section{La précision de la preuve}

7 Au niveau des faits, on utilise la preuve que l'on obtient à partir de la comparaison avec d'autres groupes - qu'il s'agisse de nations, d'organisations, de comtés ou de pavillons hospitaliers - pour vérifier que la preuve initiale était correcte. Le fait est-il vraiment un fait? Ainsi, les faits sont répétés afin de fournir des éléments comparatifs de preuve, soit de façon interne (au sein de l'étude), soit de façon externe (hors de l'étude), soit les deux. Les sociologues s'accordent généralement sur le fait que la réplication est le meilleur instrument de validation.

Bien que cet usage de l'analyse comparative ne constitue pas, en tant que tel, notre but, il est assurément subsumé sous notre but. Nous souhaitons naturellement avoir autant de certitude que possible à propos de la preuve, et nous la vérifierons aussi souvent que possible. Cependant, le fait qu'une partie des éléments probants ne soit pas entièrement vérifiée ne doit pas nous troubler outre mesure; en effet la production de théorie ne s'appuie pas sur le fait, mais sur la catégorie conceptuelle (ou une propriété conceptuelle de la catégorie) qui en a été extraite. Un concept peut être produit à partir d'un fait qui devient alors simplement un élément dans un univers de nombreux indicateurs possibles pour le concept et de données pouvant lui être associées ${ }^{4}$. On doit rechercher ces indicateurs pour l'analyse comparative.

$9 \mathrm{Au}$ cours du processus de découverte de la théorie, on produit les catégories conceptuelles ou leurs propriétés à partir d'éléments probants. On se sert ensuite de la preuve à partir de laquelle a émergé la catégorie pour illustrer le concept. La preuve n'a pas à être exacte au-delà de tout doute (elle n'a même pas à l'être dans des recherches qui ne se préoccupent que de l'exactitude), mais le concept est indubitablement une abstraction théorique pertinente à propos de ce qui a cours dans le domaine étudié. En outre, le concept lui-même ne changera pas, alors que même les faits les plus exacts changent. Les concepts voient leur contenu périodiquement réélaboré parce que d'autres objectifs relatifs à la théorie et à l'enquête ont évolué.

10 Par exemple, la notion de perte sociale ${ }^{5}$ constitue une catégorie théorique associée au soin dont sont l'objet les patients en phase terminale (il s'agit de la mesure des 
implications sociales de la perte par rapport à la famille et à la vie professionnelle). Cette catégorie affecte clairement la manière dont les infirmières s'occupent des mourants. La catégorie de "perte sociale " peut être produite soit à partir de l'observation de la manière dont les VIP obtiennent une attention spéciale dans les unités de soin intensif, soit à partir de l'observation de la négligence dont sont fréquemment l'objet les Noirs des classes populaires dans les salles d'urgence des hôpitaux de ville. Même si les éléments probants changent (ou diffèrent dans d'autres hôpitaux pour telle ou telle raison), nous pouvons être sûrs que la perte sociale est une catégorie associée au soin infirmier et nous pouvons faire des prédictions sur cette base. Nous pouvons prédire que les patients qui ont un coefficient élevé de perte sociale recevront de meilleurs soins que ceux qui sont pourvus d'un niveau très bas dans cette catégorie. Si cette prédiction est incorrecte, nous allons très probablement découvrir que des conditions structurelles ont tendu à contredire cette relation: par exemple, lorsqu'on fait apparaître comment l'équipe médicale a surmonté cette tendance socialement induite dans un type particulier d'hôpital. En bref, la catégorie théorique découverte vit jusqu'à ce qu'on ait fait la preuve théorique de sa mort pour n'importe quelle classe de données, alors que la durée de vie de la preuve exacte qui a servi à construire la catégorie peut être brève.

\section{Généralisations empiriques}

11 Un autre usage courant des études comparatives consiste à établir la généralité d'un fait. Le tabou de l'inceste existe-t-il dans toutes les sociétés? Le personnel infirmier est-il presque entièrement de sexe féminin? La recherche fondamentale est-elle le but le plus valorisé dans tous les organismes de recherche? Le degré de justesse ne constitue pas un enjeu aussi important que la mise au point de la configuration structurelle d'un fait. Pour certains sociologues et anthropologues, celle-ci devient une quête d' « universaux » - les faits et leur explication par d'autres faits - qui s'appliquent à tous les hommes indépendamment de la société ou de la culture dans laquelle ils vivent.

12 Notre but, la production de théorie, subsume également la mise au point de généralisations empiriques, parce que les généralisations ne servent pas seulement à délimiter le domaine d'application d'une théorie fondé sur des données issues du terrain (grounded theory) ${ }^{6}$; mais surtout, elles nous aident à élargir la théorie en sorte qu'elle soit appliquée avec un plus haut degré de généralité et qu'elle ait un pouvoir explicatif et prédictif plus élevé. En comparant les points de similitude et de différence entre les faits, nous pouvons produire des propriétés de catégories qui accroissent la généralité et le pouvoir explicatif des catégories.

13 Par exemple, la mort par cancer aux États-Unis a pour caractéristique de survenir dans un «contexte de rétention d'information»: alors que le personnel de l'hôpital est au courant, le patient ne sait pas qu'il va mourir. La plupart des médecins ne disent pas à leurs patients qu'ils sont en phase terminale, et ceux-ci n'ont que des indices vagues et difficiles à interpréter jusqu'à la dernière étape de l'agonie ${ }^{7}$. Dans un hôpital japonais que nous avons visité, les patients savent d'une manière caractéristique qu'ils vont mourir (il s'agit d'un contexte d'information ouverte). Pourquoi? Parce que le pavillon hospitalier est ouvertement désigné comme étant celui des cancéreux. Le patient qui entre dans le pavillon lit un indice clair qui l'informe qu'il va mourir. Alors qu'aux États-Unis les indices tendent à être vagues et fugaces, nous avons découvert à travers l'exemple japonais que ceux-ci peuvent être clairs même au tout début d'un processus qui va 
conduire à long terme à la mort. Jusqu'alors, nous ne nous étions pas rendu compte que le degré de clarté des indices peut varier au début d'une maladie comme le cancer. Nous avions pensé que des indices clairs n'émergeaient qu'en phase terminale, par exemple, quand le prêtre arrive, ou que la douleur devient intolérable, ou lorsqu'une dégénérescence physique massive survient.

Ces données comparatives en provenance du Japon nous ont fourni un stimulant pour trouver des endroits aux États-Unis où des indices clairs sont disponibles dès le début du processus qui va conduire à la mort. Nous avons constaté que, dans un hôpital pour anciens combattants et dans le pavillon hospitalier d'une prison, on offrait aux patients dès le début des indices clairs sur le fait qu'ils avaient un cancer. Nous avons ainsi découvert le fait que, dans les conditions structurelles qui sont celles des patients captifs au sein des hôpitaux contrôlés par le gouvernement, on tend à mourir dans un contexte d'information ouverte. Mais ce n'est pas le cas pour la plupart des patients aux États-Unis.

\section{La détermination d'un concept}

15 Il existe un autre usage des données comparatives, qui exige habituellement un travail minutieux et appliqué, pour déterminer une unité d'analyse pertinente pour une étude de cas. On le fait en déterminant les dimensions du concept qui désigne cette unité. Pour être certain que le lecteur va comprendre l'objet d'une monographie donnée, en comparaison avec des unités apparemment similaires, l'auteur compare cette unité avec d'autres unités. La comparaison fait ressortir les éléments distinctifs ou la nature du cas étudié. Par exemple : Cressey a comparé très minutieusement les dancings à entraîneuse avec tous les autres types de dancings avant d'avancer dans son analyse ${ }^{8}$. Lipset, Trow et Coleman ont comparé la nature politique particulière de l'ITU [International Teamsters Union] avec la structure politique particulière de tous les autres syndicats en vue de démontrer le caractère « déviant » de leur objet d'étude ${ }^{9}$. Wirth a comparé le ghetto de Chicago avec ses équivalents européens pour démontrer les changements distinctifs qui caractérisent les ghettos du Nouveau Monde ${ }^{10}$. Coleman, à l'aide de machines IBM, a soigneusement fait la distinction entre des types d'établissements secondaires selon trois dimensions, vérifiées empiriquement pour s'assurer qu'elles ne diffèrent pas seulement dans le scénario qu'écrit le sociologue ${ }^{11}$.

16 On a recours à cet usage courant (et requis) de l'analyse comparative lorsqu'on entreprend la présentation d'une étude avec l'objectif d'en venir directement à l'histoire qui s'ensuit. Un tel usage est évidemment subsumé sous l'objectif qui consiste à produire de la théorie. Cependant, quand l'objectif du chercheur consiste seulement à déterminer une unité d'analyse, il gâche ses chances d'élever sa capacité de production de théorie à un plus haut degré qu'avec n'importe quel autre usage de l'analyse comparative. Les éléments empiriques distinctifs qui permettent de délimiter des unités de comparaison sont cantonnés au niveau des données, en vue de fournir une compréhension claire des définitions différentielles. En conséquence, on prend soin de ne prêter aucune attention aux propriétés générales que les unités considérées ont en commun, lesquelles pourraient apparaître au cours de la comparaison. On ne tolère aucune ambiguïté concernant les similarités qui apparaissent entre unités en concurrence et qui indiqueraient une propriété générale sous-jacente les parcourant toutes. L'analyse comparative est ainsi soigneusement expulsée du tableau, pour éviter d'«interrompre " à nouveau le monologue. 


\section{La vérification de la théorie} invariablement de test pour ses hypothèses - et de ce fait pour la pertinence des catégories qu'il construit; les données comparatives fournissent les meilleurs tests. Implicitement et explicitement, le chercheur contrôle en permanence sa théorie alors même que les données affluent. La vérification explicite au-delà du test des hypothèses peut conduire à la démonstration de régularités majeures et d'universaux, au constat de variations stratégiques dans la théorie sous l'effet de conditions différentes ${ }^{12}$, et à des modifications fondées de la théorie ${ }^{13}$. Un peu de production théorique peut être incluse, mais le chercheur concentre son attention sur la vérification; il produit de la théorie uniquement pour modifier sa théorie initiale à partir du résultat des tests. L'essentiel de son travail s'appuie sur des théories existantes; par exemple, le travail de Blauner dans le cadre de la théorie marxienne, ou celui de Lipset dans le cadre de la théorie de Michels ${ }^{14}$.

Certains chercheurs concentrent leur attention sur la vérification de la théorie nouvelle qui émerge de leurs données ${ }^{15}$. Ainsi, dans leur travail, la théorie est produite, mais son émergence est prise comme allant de soi. Le travail porte intentionnellement sur la vérification de la théorie émergente. Les chercheurs sont préoccupés par le "contrôle " d'un « ensemble émergent de propositions ». Leur technique favorite consiste à chercher des cas négatifs ou à s'efforcer d'accumuler des cas positifs en vue d'ajouter des preuves pour leurs hypothèses. Et alors que, comme c'est le cas dans la recherche de Dalton, on peut se donner beaucoup de mal pour chercher activement des groupes à fin de comparaison, d'autres chercheurs peuvent utiliser fortuitement ou même implicitement des groupes à fins de comparaison.

Ces chercheurs ne semblent pas, dans des études particulières, s'être concentrés directement sur les conditions d'émergence de leur propre théorie. En conséquence, ils n'ont pas exploré les voies par lesquelles ils auraient pu produire plus systématiquement de la théorie, et ceci avec plus de généralité et de portée conceptuelles. Une attention exclusive portée aux tests peut ainsi facilement entraver la production d'une théorie plus englobante et plus dense. D'ordinaire, on nous présente des fragments de théorie soigneusement testés, qui rendent un compte très partiel de ce qui arrive dans la situation qui fait l'objet de la recherche. On nous présente aussi une grande quantité de preuves, couplées avec l'assurance au moins implicite qu'il y en avait une énorme quantité supplémentaire lors de la vérification - parce que la preuve est encore plus importante pour le chercheur que les moyens de vérifier comment il pouvait savoir que sa théorie était «exacte ${ }^{16}$ ». Cette attention exclusive pour la preuve permet paradoxalement aux collègues mal lunés de « regarder de haut » cette théorie, en partie ou en totalité.

\section{La production de théorie}

Alors que la vérification est la tâche principale et vitale du chercheur lorsqu'il s'agit de théories existantes, nous suggérons que son principal objectif lorsqu'il développe de nouvelles théories consiste à les produire systématiquement à partir des données de sa recherche. Bien sûr, la vérification - avec le plus haut degré possible de force probatoire est un réquisit lorsqu'on découvre et qu'on produit une théorie, mais pas au point où la 
vérification l'emporte sur la production de théorie. Ainsi, la production de théorie qui se développe à partir de l'analyse comparative subsume et assume à la fois les opérations de vérification et de description exactes, mais seulement dans la mesure où ces dernières sont au service de la production de théorie. Autrement, on est sûr qu'elles l'étoufferont. Assurément, la propension à produire de la théorie est normale, et les sociologues, professeurs aussi bien qu'étudiants, pour peu qu'ils ne soient pas des «accros» de la vérification, tendent à s'investir avec enthousiasme dans la production. Mais quand celleci n'est pas clairement reconnue comme le principal objectif d'une recherche donnée, elle peut être tuée dans l'œuf sous l'effet des critiques jumelles que constituent la preuve exacte et les hypothèses vérifiées. Ceci arrive particulièrement lorsque c'est un collègue influent ou un professeur qui émet les critiques. Le chercheur voit sa confiance détruite parce que personne ne s'aperçoit que la description exacte et la vérification ne sont pas aussi cruciales lorsque l'objectif est de produire de la théorie. C'est particulièrement vrai parce que la preuve et les tests ne détruisent jamais une théorie (quel que soit son degré de généralité) : elles se contentent de la modifier. Une théorie peut seulement être remplacée par une meilleure théorie ${ }^{17}$.

Quand la tâche vitale qui consiste à tester une théorie nouvellement produite commence, il est probable qu'on oublie ou qu'on ignore les éléments probants à partir desquels elle a été produite. L'attention se porte alors sur les nouveaux éléments de preuve qu'on va utiliser pour vérifier seulement une partie de la théorie. En outre, les sociologues jugeront utile de risquer un moment de leur carrière pour tester des théories fondées sur la recherche de terrain, puisqu'il est certain que ces théories auront un haut degré d'applicabilité dans les domaines qui font l'objet de la recherche. La situation contraste avec le risque qu'on prend lorsqu'on teste une théorie hypothético-déductive dont les relations avec le domaine de comportement qu'elle prétend expliquer sont toujours discutables, puisqu'elle a été purement élaborée en fonction de suppositions a priori et d'un zeste de sens commun, assaisonné de quelques vieilles spéculations théoriques issues de l'érudition ${ }^{18}$. Le vérificateur peut découvrir que la théorie spéculative n'a rien à faire avec les éléments de preuve dont il dispose, à moins qu'il ne ménage une connexion forcée 19.

La production de théorie offre les mêmes profits que le test de la théorie, plus un profit additionnel. La vérification d'une théorie hypothético-déductive nous fournit au mieux la reformulation d'une ou deux hypothèses et un ensemble de spéculations non confirmées. $\mathrm{Au}$ pire, nous avons une théorie qui ne semble ni convenir ni marcher (et avec peut-être le pénible sentiment qu'un " penseur » a pu nous mener en bateau). On peut utiliser une théorie fondée sur la recherche de terrain pour tester une théorie hypothético-déductive ressortissant au même domaine en comparant les deux théories de manière plus exhaustive que ce que pourrait fournir une description exacte utilisée pour vérifier quelques propositions. Qu'il y ait ou non une théorie spéculative préexistante, la découverte nous donne une théorie qui « convient ou qui marche » pour un domaine réel ou formel (bien que des tests supplémentaires, une clarification ou une reformulation soient encore nécessaires), puisque la théorie a été dérivée à partir des données, et non pas déduite de suppositions logiques.

Puisque la preuve exacte n'est pas aussi cruciale pour produire de la théorie, le type de preuve, aussi bien que le nombre de cas, ne sont pas non plus aussi cruciaux. Un cas singulier peut être l'indicateur d'une catégorie ou d'une propriété conceptuelle générale ; quelques cas supplémentaires peuvent confirmer l'indication. La production de théorie à 
partir de l'analyse comparative exige une multitude de cas soigneusement choisis, mais $i$ ne s'agit pas d'exercer une pression sur le sociologue pour qu'il "connaisse tout le terrain » ou qu'il sélectionne tous les faits «à partir d'un soigneux échantillonnage au hasard ». Sa tâche ne consiste pas à fournir une description complète d'un objet, mais à développer une théorie qui permette de rendre compte d'une partie importante des comportements considérés. Le sociologue dont l'objectif majeur est de produire de la théorie à partir du terrain n'a pas besoin de connaitre la situation concrète mieux que les acteurs qui y sont engagés (tâche au demeurant impossible). Son travail et sa formation le conduisent à faire ce que les agents ordinaires ne peuvent pas faire - produire des catégories générales, assorties de leurs propriétés, capables de rendre compte de situations et de problèmes spécifiques. Ces catégories peuvent fournir des guides théoriques pour l'action des agents sociaux ordinaires. Le sociologue introduit de ce fait de la théorie sociologique, et donc une différente perspective, dans la situation de ces agents. Cette perspective nouvelle peut leur être d'un grand secours.

Les sociologues qui considèrent que cette tâche constitue leur travail ne sont pas tourmentés (comme ceux qui s'efforcent de parvenir à des descriptions complètes) par des pensées du genre de celle-ci : « Tout le monde le sait ; pourquoi me fatiguer à faire un livre $^{20}$ ?» Ils ne sont pas non plus tourmentés par le sentiment selon lequel la description ne suffit pas: «Un bon sociologue de Chicago doit faire plus, mais quoi ${ }^{21}$ ?» Les sociologues qui s'assignent la tâche de produire de la théorie à partir des données de la recherche ont un travail qui ne peut être effectué que par le sociologue, et qui offre un produit significatif pour les agents sociaux comme pour les collègues. Les chercheurs, dans leur vaillant effort pour obtenir des faits, tendent à oublier que, au-delà de la méthodologie, l'offre distinctive de la sociologie à notre société est la théorie, et pas seulement une description appuyée sur de la recherche ${ }^{22}$. En fait, les organismes de collecte des données, qu'ils soient privés ou publics, sont supérieurs à n'importe quel sociologue pour ce qui est des descriptions basées sur la recherche à partir de leurs ressources propres. Le sociologue peut apporter une aide à ces organismes en leur fournissant une théorie qui rendra leur recherche pertinente. Un bref coup d'œil jeté aux rapports basés sur des collectes de données et sur des études de marché suffit à montrer l'étendue des besoins de pertinence sociologique, à la fois pour comprendre l'«amas poussiéreux» de données empilé par ces organismes et pour corriger l'idéologie conventionnelle qui guide cette accumulation de données ${ }^{23}$.

\section{NOTES}

1. Ce texte est un extrait du chapitre 2 (Generating Theory) du livre de Barney G. Glaser et d'Anselm L.Strauss, The Discovery of Grounded Theory: Stratégies for Qualitative Research, Hawthorne, Aldine de Gruyter, 1967, p. 21-31. [NdlR.]

2. Deux ouvrages ont été publiés en 1992: le premier, Miroirs et masques. Une introduction à l'interactionnisme (Paris, Métailié), est la traduction d'un ouvrage centré sur la notion d'identité dont la première édition remonte à 1959. Anselm Strauss revient sur son parcours sociologique dans une introduction originale à l'édition française. Le second, La trame de la négociation. 
Sociologie qualitative et interactionnisme (Paris, L'Harmattan), est une collection d'articles et d'extraits d'ouvrages précédé d'une substantielle introduction d'Isabelle Baszanger. Parmi les principaux ouvrages non traduits à ce jour on peut citer : Awareness of Dying (avec Barney Glaser), Chicago, Aldine, 1965; Status Passage (avec Barney Glaser), Chicago, Aldine, 1971; Qualitative Analysis for Social Scientists, New York, Cambridge University Press, 1987. [NdlR.]

3. B. G. Glaser \& A. L. Strauss, Awareness of Dying, Chicago, Aldine Publishing Co, 1965.

4. Nous appliquons ici la règle de «l'interchangeabilité des indices " proposée par Lazarsfeld en lui appliquant une nouvelle connexion. Voir P. F. Lazarsfeld \& W. Thielens, The Academic Mind, New York, Free Press of Glencoe, 1958, p. 402- 407.

5. Pour une explication et une discussion théorique de cette notion, voir B.G. Glaser \& A. L. Strauss, « The Social Loss of Dying Patients ", American Journal of Nursing, 64, 1964, p. 119-122. 6. La notion de "grounded theory " est centrale dans le travail épistémologique de Glaser et Strauss. "Grounded » désigne le fait que la théorie doit être fondée sur les données issues du terrain. Isabelle Baszanger propose de la traduire précisément par «théorie fondée ». Dans ce numéro, Jean-Pierre Olivier de Sardan propose très subtilement «modèle interprétatif issu du terrain » [N.d.T.].

7. B. G. Glaser \& A. L. Strauss, Awareness of Dying, op. cit., chap. 3 et 8.

8. P. Cressey, The Taxi-Dance Hall, Chicago, University of Chicago Press, 1932.

9. S. M. Lipset, M. Trow \& J. S. Coleman, Union Democracy, New York, Free Press of Glencoe, 1956.

10. L. Wirth, Le Ghetto [1962], Grenoble, Presses universitaires de Grenoble, 1980.

11. J. S. Coleman, The Adolescent Society, New York, Free Press of Glencoe, 1961.

12. Par exemple, R. Blauner, Alienation and Freedom, Chicago, University of Chicago Press, 1964.

13. Voir R. K. Merton, Social Theory and Social Structure, New York, Free Press of Glencoe, 1957, chap. 3.

14. Voir R. Blauner, op. cit., et M. Lipset et al., op. cit.

15. Voir par exemple M. Dalton, Men Who Manage, New York, John Wiley \& Sons, 1959, et H.S. Becker, E. Hugues \& A. L. Strauss, Boys in White, Chicago, University of Chicago Press, 1961.

16. H. S. Becker et al. (ibid.) évoquent « 5000 pages dactylographiées à simple interligne » de notes de terrain et d'entretiens transcrits (p. 30) et Dalton (ibid.) affirme que sa recherche « s'est poursuivie pendant une décennie». Ils impliquent qu'on ne peut douter de notions et de découvertes basées sur de telles quantités de temps et de preuve.

17. C'est la découverte de base faite par Thomas S. Kuhn, La structure des révolutions scientifiques [1962], Paris, Flammarion, 1976. Nous pensons que cette découverte s'applique plus aisément à une théorie fondée sur les données du terrain que sur une théorie hypothético-déductive.

18. Par exemple, dans son livre, appuyé sur une théorie à base de conjectures, Blau remarque : «L'idée et l'analyse présentées dans ce livre ont été fortement influencées par les travaux d'autres chercheurs en sciences sociales, et on en trouvera souvent la source originale dans les intuitions sur la vie sociale qu'offrent les philosophes sociaux et les penseurs du passé. » (P. Blau, Exchange an Power in Social Life, New York, John Wiley \& Sons, 1964, p. VII).

19. Le chercheur peut forcer cette connexion parce qu'on lui a appris que la science consiste à appliquer un cadre analytique à un domaine d'étude. Ne pas recourir au forçage revient alors à trahir la science. «À moins que le chercheur soit extrêmement précautionneux, il va se trouver très probablement dans la situation de trahir ses hypothèses originelles de travail, puisqu'il est contraint d'aller " là où les données le conduisent " ", met en garde un chercheur à propos du refus de l'harmonisation forcée des hypothèses avec les données. Voir S. H. Udy Jr, «Cross Cultural Analysis : A Case Study », in P. Hammond, ed., Sociologists at Work, New York, Basic Books, 1964, p.174-175. Ou bien il peut forcer la connexion pour assurer sa promotion dans une organisation peuplée de collègues qui estiment que ce type de relation devrait exister, parce qu'un « grand homme » a dit qu'il en existait une. Il va sans dire que nous croyons que le forçage 
de la connexion entre la théorie et les données est entièrement contraire à notre insistance sur le type de lien qui existe entre l'une et les autres.

20. B. Geer, « First Days in the Field », in P. Hammond, op. cit., p. 292.

21. D. Riesman \& J.Watson, «The Sociability Project: A Chronicle of Frustration and Achievement ", in P. Hammond, op. cit. p. 292.

22. Nous sommes entièrement d'accord avec Zetterberg sur le point de savoir si la sociologie progressera plus en se concentrant sur la théorie ou sur la méthodologie. Mais nous sentons qu'une méthodologie de la production est nécessaire pour le progrès théorique. Voir H. L. Zetterberg, On Theory and Verification in Sociology, Towota, Bedminster Press, 1963, "Préface».

23. La pertinence sociologique des vastes recueils de statistiques gouvernementales concernant les différences de soin médicaux en fonction de la stratification sociale fournit ici un bon exemple. La signification de sens commun de ces statistiques est évidente, mais une interprétation sociologique plus profonde ne guide pas ces enquêtes, pas plus qu'elle n'affecte les politiques publiques. Le savoir sociologique à propos des styles de vie socio-économiques et de l'organisation de l'offre médicale peut être facilement utilisé pour éclairer les données officielles. Voir le texte concernant la politique de santé rédigé par Anselm Strauss pour l'Institute for Policy Studies (Washington, D.C., juillet 1965).

\section{RÉSUMÉS}

Cet extrait du chapitre 2 de l'ouvrage classique de Barney Glaser et Anselm Strauss, The Discovery of Grounded Theory (1967) met en valeur l'intérêt des propositions épistémologiques de la sociologie interactionniste. En restant toujours au plus près des exigences spécifiques de l'observation réglée du monde social, Glaser et Strauss indiquent les impasses auxquelles conduisent les problématiques de la validation d'une théorie par les tests empiriques, comme celle de Merton: les sciences sociales qui ne satisfont pas aux conditions logiques de la falsification obéissent à un autre registre de la production théorique. Cet extrait présente ainsi la notion de grounded theory à partir d'une réflexion sur les usages de l'analyse comparative considérée comme une méthode de portée générale, au même titre que les méthodes expérimentale et statistique. C'est au cours du processus de découverte de la théorie que sont produites les catégories conceptuelles ou leurs propriétés à partir d'éléments probants. La preuve à partir de laquelle a émergé la catégorie est utilisée en retour pour illustrer le concept, dont le contenu est périodiquement réélaboré, selon l'évolution des objectifs relatifs à la théorie et à l'enquête.

This extract of chapter 2 of the classic work of Barney Glaser and Anselm Strauss, The Discovery of Grounded Theory (1967), highlights the importance of epistemological proposals of interactionist sociology. Remaining as close as possible to the specific requirements of regular observations of the social world, Glaser and Strauss point out the dead ends into which the problematics of the validation of a theory by empirical tests lead, such as that of Merton : social sciences which do not satisfy logical conditions of "falsification" obey another system of theoretical production. This extract thus presents the notion of "grounded theory" from a reflection on the uses of comparative analysis considered as a method generally accessible, on the same grounds as experimental and statistical methods. In the process of discovery of the theory, conceptual 
categories or their properties from conclusive elements were produced. The evidence from which the category emerged is in turn used to illustrate the concept whose content is periodically reelaborated, according to the evolutions of the objects related to the theory and to the survey. 